\title{
What did the bronze eye-like prism tell us?
}

\author{
HE RongDing $^{1 *} \&$ HE RongQiao ${ }^{2}$ \\ ${ }^{1}$ The Fine Arts College, Chengdu University, Chengdu 610016, China; \\ ${ }^{2}$ Institute of Biophysics, Chinese Academy of Sciences, Beijing 100101, China \\ Received January 15, 2014; accepted February 26, 2014; published online March 14, 2014
}

Citation: He RD, He RQ. What did the bronze eye-like prism tell us? Sci China Life Sci, 2014, 57: 445-447, doi: 10.1007/s11427-014-4634-1

In 1986, the Sanxingdui ("Three-Star Mound") Relics were discovered in Guanghan City of Sichuan Province in China. Two major sacrificial pits were excavated and thousands of treasures were discovered, including bronze artifacts, jade wares and potteries. These relics have unveiled the history of the ancient Shu Kingdom during the era of the Shang Dynasty, which ruled about 3000-4000 years ago [1]. Amongst the bronze artifacts were human heads (13 from Pit 1, and 44 from Pit 2) and faces (one from Pit 1 and 20 from Pit 2) with their eyes divided along a dorsoventral borderline, suggesting that the people of the Shu Kingdom had a unique point of view on human eye.

The bronze eye-like prism is one of the most striking characteristics of the artifacts. Three different styles of bronze eye-like prisms have been identified (Figure 1): an intact eye-like prism with a borderline between the dorsal and ventral areas (Figure 1A); a split-joint prism with dorsoventral sections (Figure 1B); and a split-joint prism with dorsoventral and nasotemporal sections (Figure 1C). The construction of the bronze eye-like prism is comparable to the human eye in that the structure of the human eye is divided into dorsoventral and nasotemporal nervous fiber projections [2]. Furthermore, as the structure of the eye-like prism is much larger than the eye of bronze head and the real human eye, it suggests the prism is also representative of something other than structure.

The bronze eye-like prism has been said to also reflect the true nature of the human visual field [3]. To investigate this possibility further, we compared the features of the

*Corresponding author (email: 572522845@qq.com) prism to that of the human visual field. As shown in Figure 1E, Stanojcic and colleagues [4] have analyzed the human visual field using the Humphrey Field Analyzer II (HFA; Humphrey Systems, Dublin, CA, USA) and have demonstrated that the true visual field of binocular vision is similar to a prism. In fact, humans can perceive an approximate prism-like visual field when the eyes are immobile and looking directly forward. This suggests that both the intact and the split-joint bronze eye-like prism represent human vision and visual field, in particular the true binocular visual field.

The visual field can be described as the space or range within which objects are visible to the immobile eyes at a given time. Vision can be lost, and the extent of the visual field can be diminished, when one's visual system has gotten lesions. This condition, termed hemianopia or hemianopsia, is a type of anopsia where loss of vision or blindness takes place in half the visual field of one or both eyes, and in most cases this loss respects the vertical midline. The most common causes of this damage include brain trauma, stroke and tumor, which result in lesions of the visual nerves [5]. Lesions at specific parts of the visual system cause defects in the nerve projections of the visual field (Figure 2). We postulate that, in addition, the split-joint bronze eye-like prism represents the human visual field as occurs in hemianopia, the loss of partial vision (Figure 2; Supplementary data 1).

Our views on the eye-like prism are based on a number of key observations. First, it is evident from Sanxingdui Relics that the ancient Shu people adored the eye for hundreds of years and paid great attention to its structure and function. Second, the Shu people named their capital city "Qu-Shang", which literally means "a pair of superior eyes" (Figure 3). To us, this demonstrates that the people of the 

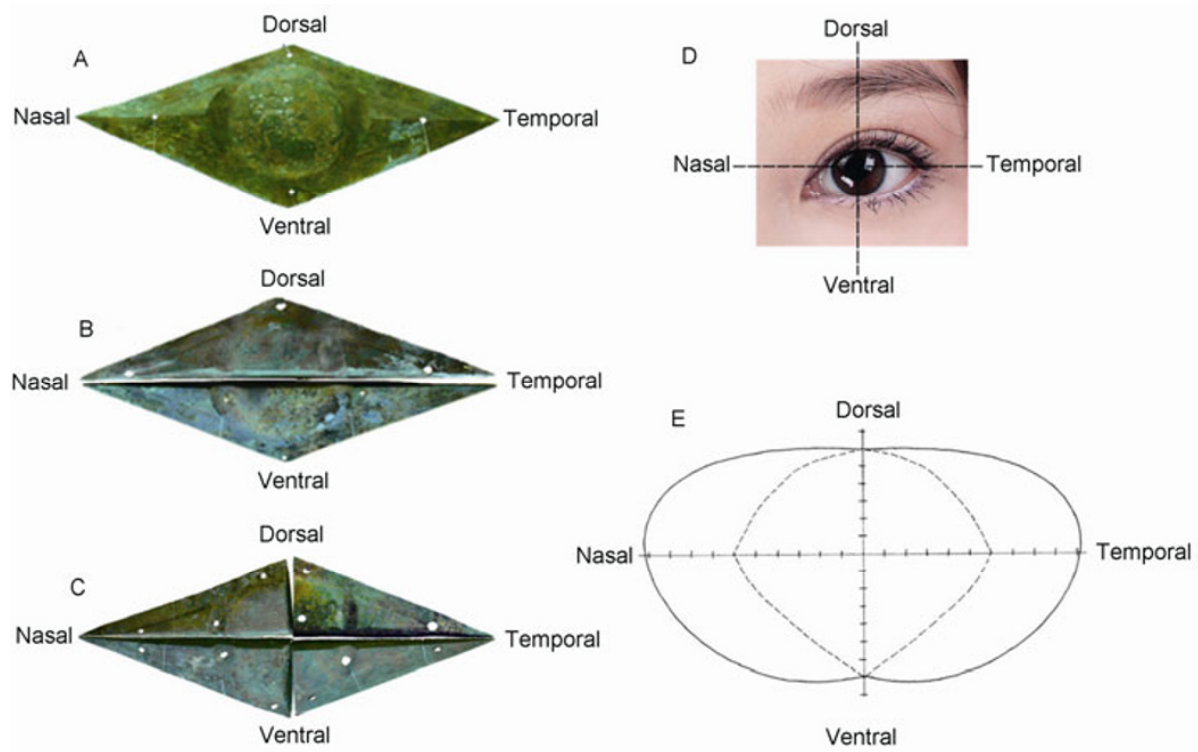

Figure 1 The bronze eye-like prisms excavated from Sanxingdui. Several bronze eye-like prisms were found in sacrificial Pit 2 in Sanxingdui. A, An intact eye-like prism with dorsoventral borderline $(56.9 \mathrm{~cm} \times 23.3 \mathrm{~cm})$. B, A split-joint prism with dorsoventral detached sections $(55.6 \mathrm{~cm} \times 12.5 \mathrm{~cm}$ each). C, A split-joint prism with dorsoventral and nasotemporal sections $(29.2 \mathrm{~cm} \times 12.4 \mathrm{~cm}$ each). D, The human eye is organized along both a dorsoventral axis and a nasotemporal axis, with each containing different molecular components and nerve fiber projections. E, The solid line delineates the normal extent of human binocular visual field, whereas the dashed line delineates approximation of the true binocular visual field. In humans, the true visual field, which contains dorsoventral and nasotemporal components, looks like a prism [4].

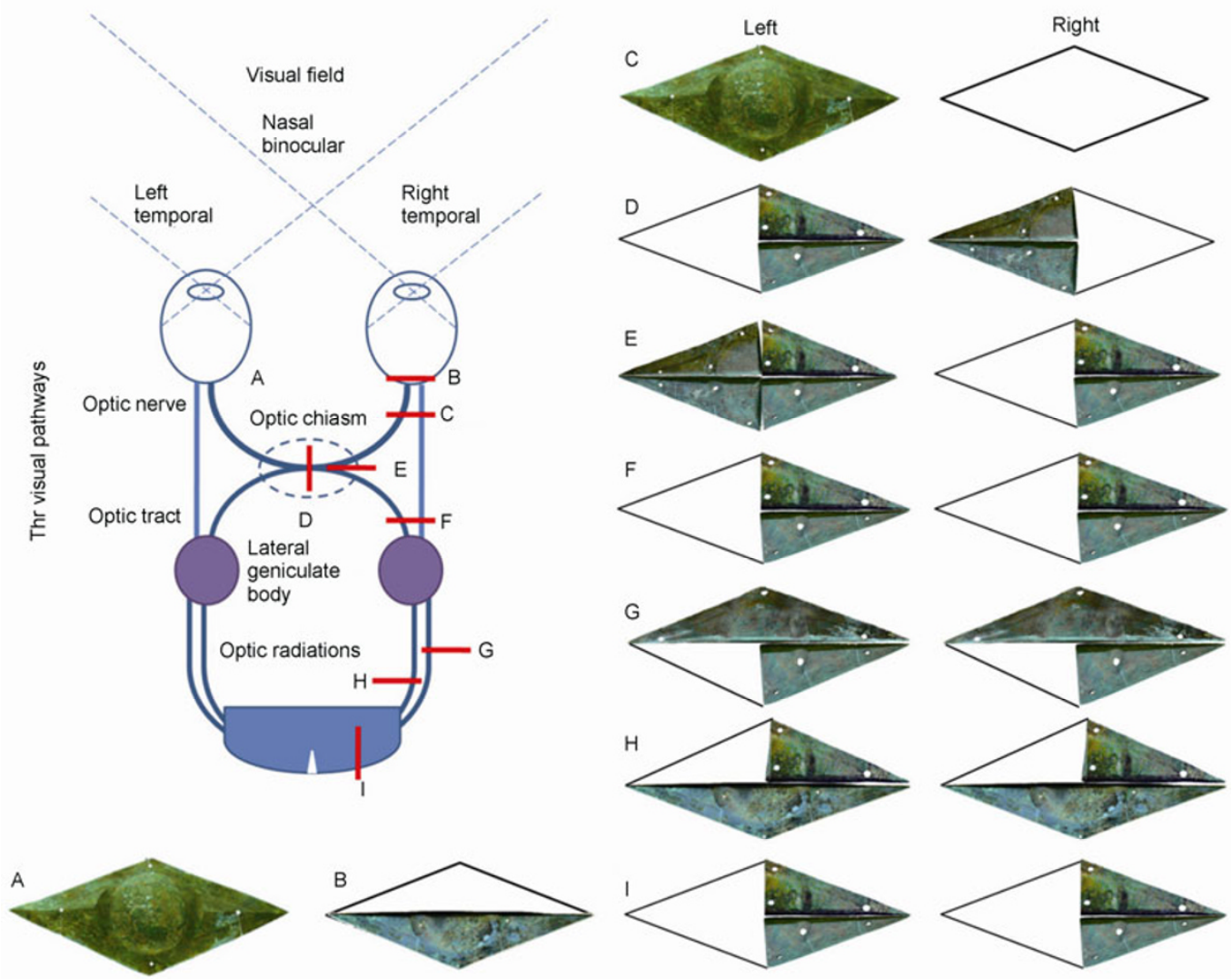

Figure 2 Human hemianopsia showed in bronze eye-like prism vision. The bronze eye-like prism displays physical similarities to human hemianopsia. A, Normal eye. B, Unilateral defect found with arterial occlusion branch retinal vein thrombosis inferior retinal detachment. C, Total blindness of right eye due to complete lesion of right optic nerve. D, Bipolar hemianopia due to midline chiasmal lesion. E, Right nasal hemianopia due to lesion involving right perichiamal area. F, Left homonymous hemianopia due to lesion or pressure on right optic tract. G, Left homonymous inferior quadrantanopia due to involvement of lower right optic radiations. H, Left homonymous superior quadrantanopia due to involvement of upper right optic radiations. I, Left homonymous hemianopia due to lesion of right occipital lobe (Supplementary data 1 and 2 in Supporting Information). The bronze, black and red lines represent vision, blindness and lesion, respectively. 
ancient Shu kingdom believed in the eye and thus had an appreciation that allowed them to learn and understand more about the eye than their contemporaries. Third, the human binocular visual field is not a circle, but rather a prism-like field that is similar to the shape of the eye-like prism. Fourth, it is likely that during this era hemianopia was caused by direct brain trauma, rather than by stroke or tumor [5], because during the era of the Yinshang dynasty, the average lifespan was approximately 34.3 years (Wang and Yang. Chin Cult Relics News, 2009, 1/Sept: pp.7) and thus the incidence of stroke and tumor was likely low. Fifth, during battle in the Neolithic and Bronze Ages it was common to aim weapons such as bronze stagger-axes, arrows and sharp stones at the human head with the intention of damaging the skull and brain. These weapons were capable of causing direct and penetrating trauma, which often led to damage of the brain, including the visual nerves. Though officers of that era often carried bronze swords into battle,

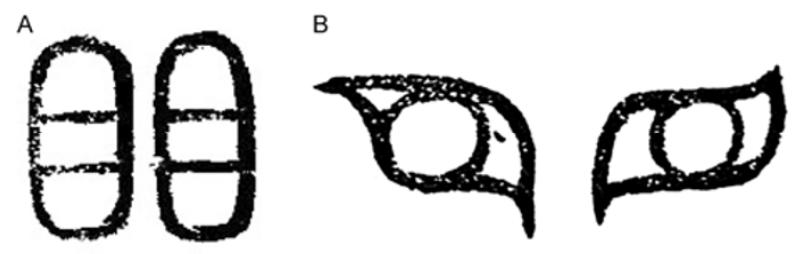

Figure 3 The ancient Chinese character for $\mathrm{Qu}$. The name for the capital city of the ancient Shu is Qu-Shang, where Qu means "the binocular vision" (Words and Expression, by Xushen, Han dynasty, reprinted in 1877) (A) and also "a pair of eyes" (Collation and Explanation, reprinted in 1883) and Shang means "superior" (B). Thus, Qu-Shang can be translated to mean "a pair of superior eyes". these were quite fragile and were often ornamental; thus, brain trauma that included the visual system was likely to be the most commonly sustained injury during the Bronze Age. Sixth, it was not until after the collapse of the ancient Shu kingdom that the long iron was introduced into battle. The weapon was designed as a mode of operation to pierce the chest, abdomen and arteries. As a result, the incidence of brain injury on the battlefield in the Bronze Age of the ancient Shu Dynasty must have been much higher than in the Iron Age. We therefore believe that the people of the Shu kingdom had many opportunities to see and learn the symptoms of hemianopia.

We suggest that the bronze eye-like prism, especially the split-joint relics, are particularly important artifacts as they may be the earliest bronze object demonstration that accurately represents the structure of the human eye, the visual field, and conditions such as hemianopia.

1 He RD, Chen C, Zhang SS, He YH, Wei Y, Yang Y, Li DS, Bao XF, He RQ. A probable copper resource for Sanxingdui bronze artifacts. Adv Edu Res, 2013, 18: 342-354

2 He RD, Liu MN, Liu Y, Zhang SD, He RQ. A coincidence between a natural mosaic eye and a bronze eye found in Sanxingdui. J Life Sci, 2008, 2: 53-57

3 He RD, Zhou J, Zhang SS, Yang Y, Wei Y, He YH, Liu Y, He RQ. Investigation of the characteristic of Sanxingdui bronze artifacts based on eye construct. Adv Edu Res, 2013, 18: 392-400

4 Stanojcic N, Wilkins M, Bunce C, Ionides A. Visual fields in patients with multifocal intraocular lens implants and monovision: an exploratory study. Eye, 2010, 24: 1645-1651

5 O’Neill E, O’Connor J, Brady J, Reid I, Logan P. Prism therapy and visual rehabilitation in homonymous visual field loss. Opt Vis Sci, 2011, 88: 263-268

Open Access This article is distributed under the terms of the Creative Commons Attribution License which permits any use, distribution, and reproduction in any medium, provided the original author(s) and source are credited.

\section{Supporting Information}

\section{Supplementary data 1 Hemianopia-definition of hemianopia}

http://medical-dictionary.thefreedictionary.com/hemianopia

Supplementary data 2 Medical notes, ophthalmology articles: optic pathway

http://www.fastbleep.com/medical-notes/surgery/20/100/716

The supporting information is available online at life.scichina.com and link.springer.com. The supporting materials are published as submitted, without typesetting or editing. The responsibility for scientific accuracy and content remains entirely with the authors. 